\title{
PROGRAMAS DE SALUD Y PRÁCTICAS DE CUIDADO NUTRICIONAL INFANTIL
}

\author{
HEALTH PROGRAMS AND CHILD NUTRI- \\ TIONAL CARE PRACTICES
}

\section{PROGRAMAS DE SAÚDE E PRÁTICAS DE CUIDADOS DE NUTRIÇÃO INFANTIL}

\section{Resumen}

Objetivo: Analizar las prácticas de cuidado nutricional consideradas por programas y planes de salud implementados en la ciudad de Córdoba, especialmente en familias en situación de pobreza.

Método: Investigación cualitativa de análisis documental basado en el análisis de fragmentos de discurso según Jäger, teniendo en cuenta aspecto semántico y contextual. Resultados: En los documentos analizados se incorpora la perspectiva de derechos, el cuidado es considerado una condición previa al cumplimiento de los derechos. Hacen referencia a normas nacionales y/o provinciales, incorporan actividades asistenciales y comunitarias, pretendiendo incorporar la mirada de la comunidad; así como la comunicación entre efectores y cuidadores.

Scruzzi Graciela Fabiana ${ }^{1}$ Lucchese Marcela²

'Lic. en Nutrición, Especialista en Epidemiologia, Magister en Salud Pública. Docente de la Universidad Nacional de Córdoba y de la Universidad Católica de Córdoba, Argentina. Correo gracielafabiana@hotmail.com 2Doctora en Ciencias de la Salud. Licenciada y Profesora en Ciencias de la Educación.

Docente de la Universidad Nacional de Córdoba y de la Universidad Católica de Córdoba, Argentina

Trabajo recibido: 02 de Mayo 2017 Aprobado: 01 de Agosto 2017.
Conclusiones: Si bien en la Argentina las políticas de salud se conciben desde el régimen familista, hay un reconocimiento de la necesidad de empoderar a las familias para el ejercicio del derecho al cuidado, en todos los documentos se proponen actividades en un marco de diálogo entre efectores y cuidadores.

Palabras clave: programas de salud, cuidados nutricionales, infancia.

\section{Abstract}

Objective: To analyze nutritional care practices considered by health programs and plans implemented in the city of Cordoba, particularly in families living in poverty. Method: Qualitative research of document analysis based on discourse fragment analysis according to Jäger, considering semantic and contextual aspects.

Results: The perspective of rights is incorporated in the 
analyzed documents, care is considered a previous condition to the fulfillment of rights. They refer to national and/or provincial norms, incorporate care and community activities, pretending to incorporate the community outlook, as well as the communication between effectors and caregivers.

Conclusions: Although health polices in Argentina are conceived from the family based regime, there is understanding of the need to empower families in exercising their right to care; in all the documents, activities are proposed within a framework of dialog between effectors and caregivers.

Key words: health programs, nutritional care, childhood.

\section{Resumo}

Objetivo: Analisar as práticas de cuidados nutricionais consideradas pelos programas e planos de saúde implementados na cidade de Córdoba, especialmente nas famílias que vivem na pobreza.

Método: pesquisa qualitativa de análise documental baseada na análise de fragmentos de fala de acordo com Jäger, levando em consideração aspectos semânticos e contextuais.

Resultados: nos documentos analisados, a perspectiva dos direitos é incorporada, o cuidado é considerado uma condição prévia ao cumprimento dos direitos. Referem-se a normas nacionais e / ou provinciais, incorporam atividades comunitárias e de cuidados, visando incorporar a perspectiva da comunidade; bem como a comunicação entre efetores e cuidadores.

Conclusões: Embora as políticas de saúde na Argentina sejam concebidas a partir do sistema familiar, há reconhecimento da necessidade de capacitar as famílias para exercer o direito à assistência, em todos os documentos, as atividades são propostas dentro de um quadro de diálogo entre efetores e cuidadores.

Palavras chave: Programas de Saúde, Cuidados Nutricionais, Infância.

\section{Introducción}

A Kraft y Furlong (2006) plantean que una política pública es un curso de acción o de inacción gubernamental, en respuesta a problemas públicos (1).Castro (1998) señala que las políticas públicas carecen de neutralidad ideológica y política, son dialécticas e históricas, se construyen sobre definiciones específicas, de sociedad, de familia, de necesidades sociales, de propiedad, de trabajo, de control-coerción y de mujer-hombre, entre otros. Implican intereses diversos: de clase, de raza, de etnia, de género, de edad, de ubicación geográfica: regionales, nacionales y/o internacionales, económicos, políticos, culturales, educativos, y exponen una visión específica sobre la realidad, los problemas y necesidades sociales y las maneras en que éstos deben ser atendidos o resueltos (2).

Si bien las políticas públicas se concretan a través de distintas normas, instituciones o programas públicos diseñados y desarrollados por diversos actores de la sociedad, siempre son validadas por los poderes del Estado.

Grassi (2003), considera que el Estado expresa y produce un orden político social cuando se activan políticas; convirtiéndose en actor y ámbito de la producción de los problemas sociales, en la delimitación de su propia responsabilidad en la definición de los sujetos merecedores de sus intervenciones y las condiciones para dicho merecimiento (3).

En el marco de esta investigación interesa conceptualizar la política social como el conjunto de directrices, orientaciones, criterios y lineamientos conducentes a la preservación y elevación del bienestar social, procurando que los beneficios del desarrollo alcancen a todas las capas de la sociedad con la mayor equidad (4).

Los procesos de reforma del Estado desarrollados en la última década en Argentina modi- 
ficaron la correlación de fuerzas, el alcance y el modo de intervención estatal, lo que implicó una redefinición de los roles y prioridades del Estado, y en la forma de relacionarse con la sociedad civil. Tales cambios se evidenciaron en el diseño y la implementación de las políticas públicas y en las políticas sociales, influyendo en los contenidos o las temáticas que ingresan a la agenda estatal, en las formas de resolución y en tipo de soluciones. Por ello una forma de visualizarlos es por medio del análisis de los objetivos, el diseño y las prácticas de gestión de las políticas públicas, asimismo se pueden visualizar por medio del análisis de los diversos actores que participan en dichos procesos y del modo que interactúan y se relacionan en esos marcos institucionales específicos.

Estas reformas afectaron tanto a los marcos institucionales como a la configuración del mapa de actores relevantes que interactúan alrededor de las políticas públicas. Por ello para analizar las políticas públicas es necesario considerar la presencia plural de actores políticos y sociales e internacionales (5).

En Argentina, el sistema de salud se subdivide en sector privado, sector de la seguridad social a través de las obras sociales y sector público. En cuanto a la cobertura de salud en la provincia de Córdoba, según el censo provincial 2008, el 66\% (2.139.701) de los habitantes de la provincia de Córdoba cuenta con cobertura de salud (obra social y/o prepaga), por lo tanto, el 34\% de la población de la Provincia posee únicamente cobertura mediante el sistema público de salud (6).

El sistema público de salud está integrado por los hospitales públicos y los centros de atención primaria de la salud que funcionan bajo la coordinación de Ministerios y Secretarías de la Salud de las diferentes jurisdicciones (nacional, provincial o municipal) y presta servicios de provisión gratuita.

Los sistemas locales de salud, fueron propuestos por la OPS como una manera de aplicar la estrategia de atención primaria de la salud y cumplir con los principios y objetivos de la meta de Salud para todos. Para cumplir esta misión, un sistema local y la red de servicios, debe tener un conocimiento preciso de las necesidades de la población y los recursos disponibles, con el fin de definir las acciones que sean necesarias emprender para responder a las realidades locales de salud (7).

Toda política social tiene como supuesto una organización social del cuidado que define a los/as actores sociales asignándoles responsabilidades.Desde un punto de vista estructural, la forma en que se organizan los sistemas de salud, educación y previsión social, tiene profundas implicaciones en cuanto a la organización del cuidado social (8).

Las investigaciones realizadas principalmente en los países de la Unión Europea introdujeron una aproximación de género en un campo que ignoraba esta dimensión: como es el de las políticas sociales y los Estados de bienestar. Se ha mostrado que el carácter doméstico de los cuidados ha sido la base para la exclusión de las mujeres de los derechos ciudadanos propugnando un concepto de ciudadanía social que reconozca la importancia de los cuidados y las responsabilidades domésticas para la sociedad (9).

La división del cuidado entre estado, familia, mercado y comunidad se diferencia según la orientación de las políticas públicas (familista o desfamiliarizadora). En el régimen familista típico la responsabilidad principal del bienestar corresponde a las familias y a las mujeres en las redes de parentesco. En el régimen desfamiliarizador hay una derivación hacia las instituciones públicas y hacia el mercado.

Autores como Letablier (2007) y Aguirre (2007) consideran que las problematizaciones en torno a las prácticas y relaciones de cuidado deben analizarse en las intersecciones del espacio doméstico con las políticas públicas y con otras formas mercantilizadas o comunitarias de cuidado que tienen lugar en nuestra sociedad $(9,10)$.

En Argentina, históricamente las políticas estatales se complementaron con las acciones desarrolladas por las familias en el marco de la consolidación del modelo de familia nuclear. Así la atención de las necesidades de cuidado recayó principalmente sobre las unidades domésticas consolidando un modelo familista y feminizado de cuidado infantil, en tanto 
las prácticas de cuidado fueron asumidas principalmente por las familias y al interior de las mismas fundamentalmente por las mujeres $(11,12)$. Es importante "desprivatizar" este tema, sacarlo de la esfera privada y convertirlo en un tema de interés público para integrarlo a la agenda pública de los problemas sociales.

En relación a la implementación de las políticas en la niñez, el Estado argentino ha avanzado en el reconocimiento del derecho de los niños y las niñas al cuidado y la educación desde temprana edad.En el año 1994 la Argentina incluye en su Constitución los siguientes tratados internacionales: la Convención de los Derechos del Niño (CDN), y la Convención sobre la Eliminación de Todas las Formas de Discriminación contra la Mujer (CEDAW). En el año 2005, se promulga La Ley Nacional 26.061 de Protección Integral de los Derechos de las Niñas, Niños y Adolescentes, en consonancia con la CDN, la cual crea un sistema de protección de los derechos de todos los niños, niñas y adolescentes del país, en cuya base se encuentra el conjunto de políticas públicas básicas y universales para el pleno desarrollo de los chicos en todas las áreas; y define las responsabilidades de la familia, la sociedad y el Estado en relación con esos derechos. Esta ley en su artículo $14^{\circ}$ garantiza el derecho a la salud integral, provocando un cambio de paradigma, desplazando la idea de niño como objeto de tutela por un nuevo concepto de niño como sujeto de derecho desde el nacimiento (13).

El desarrollo de la primera infancia, en particular el desarrollo físico, socioemocional y lingüístico-cognitivo, determina de forma decisiva las oportunidades en la vida de una persona y la posibilidad de gozar de buena salud, pues afecta la adquisición de competencias, la educación y las oportunidades laborales. A través de esos mecanismos y de forma directa la primera infancia influye en el riesgo posterior de obesidad, malnutrición, problemas de salud mental, enfermedades cardiacas y delincuencia. Al menos 200 millones de niños en el mundo no se desarrollan plenamente. Eso tiene enormes consecuencias para su salud y para la sociedad en su conjunto.

Desde Unicef el cuidado se define como la provisión a nivel familiar y comunitario de tiempo, atención y apoyo para satisfacer las necesidades físicas, mentales y sociales de los niños y niñas en crecimiento y otros miembros del hogar. La ingesta de nutrientes, la salud y el desarrollo cognitivo y psicosocial de niños/as se ven afectados particularmente por el conjunto de comportamientos implementados por los cuidadores para la satisfacción de estas necesidades (14).

Las prácticas alimentarias de los niños y niñas de menores de 5 años de edad, dependen en gran medida de los cuidadores nutricionales - que son casi exclusivamente las mujeres, madres -responsables de la selección, preparación y distribución de los alimentos al interior del hogar y del establecimiento de las pautas que se ponen en juego en el momento mismo de la alimentación, acompañadas en muchos caso por la familia extendida (abuelo/ as, tío/as, hermano/as) y en menor medida por organizaciones o instituciones del entorno próximo (14).

Según lineamientos de FAO (Organización de las Naciones Unidas para la Alimentación y la Agricultura) las prácticas de cuidado nutricional constituyen el menos estudiado de los tres factores subyacentes que determinan la ingesta de nutrientes y la salud y, por tanto, la supervivencia infantil, el crecimiento y el desarrollo, según el modelo conceptual de Unicef. Para los otros dos factores subyacentes seguridad alimentaria familiar y salud, existen numerosas investigaciones que los vinculan con el estado nutricional (14).

Desde fines de la década del ochenta, Unicef ha difundido ampliamente la importancia del cuidado, entendido como el vínculo entre el niño y sus padres o cuidadores, concibiendo que la primera infancia es altamente dependiente de los cuidados familiares; éstos deben tener garantizados el acceso a los recursos de conocimiento, información, tiempo y bienes materiales básicos (14).

El trabajo de cuidado, no remunerado privado, que realizan generalmente las mujeres, no es considerado como una contribución al desarrollo económico y social. Por ello, no es 
tomado en cuenta cuando se diseñan políticas públicas ni es reconocido para el acceso a la protección social de quienes lo realizan. Si bien las mujeres han logrado mayor autonomía, al conquistar progresivamente mayores espacios en su ingreso y permanencia en el mundo laboral, se produce un vacío que dificulta la organización de los tiempos del trabajo remunerado y no remunerado basado en la división sexual del trabajo tradicional, aún predominante, que requiere que las mujeres compensen la insuficiencia de los servicios públicos y los efectos desgastantes del trabajo remunerado, con su propio trabajo (15).

Es necesario incorporar una lógica de derechos a la complejidad del cuidado, no solo como beneficiario de una política de cuidado, sino como un principio inherente al ser humano. La institucionalidad vigente fortalece el modelo según el cual la responsabilidad sobre el cuidado del hogar y de los hijos sigue recayendo más fuertemente sobre las mujeres que sobre las parejas, a medida que las mujeres logran sortear las desigualdades y las discriminaciones en el mundo público, a partir de planes de igualdad de oportunidades, no se ha avanzado en considerar nuevas formas jurídicas de regular el cuidado (16).

Por todo lo antepuesto, se plantea necesario indagar acerca de los programas de salud destinados a la infancia, y si éstos incorporan las prácticas de cuidado nutricional infantil. En la indagación de los antecedentes se vislumbra la ausencia en la región de una política pública de cuidado y una postura desde los gobiernos en torno al cuidado como responsabilidad fundamentalmente de los hogares (16).

Desde una perspectiva teórica del cuidado como responsabilidad de las políticas públicas donde éstas intenten asegurar el cumplimiento y reparto equitativo de la prestación de cuidado, tanto a nivel de los hogares como de las instituciones, los interrogantes iníciales fueron:

¿Se contempla la Ley 26061 de Protección Integral de los Derechos de los niños, niñas y adolescentes en la formulación de programas y planes de salud?

¿Son consideradas las prácticas de cuidado nutricional infantil en los programas y planes de salud implementados en la Ciudad de Córdoba?

¿Cuáles son las prácticas de cuidado nutricional consideradas por los programas y planes de salud implementados en la ciudad de Córdoba?

\section{Objetivo}

Analizar las prácticas de cuidado nutricional consideradas por los programas y planes de salud implementados en la ciudad de Córdoba, especialmente en familias en situación de pobreza.

\section{Metodología}

Se trata de un estudio de carácter cualitativo que permitió explorar el sentido de las prácticas de cuidado nutricional en los programas y planes de salud implementados en la ciudad de Córdoba, especialmente en familias en situación de pobreza (17).

Para ello se identificaron los programas y planes de salud (Documentos) implementados en la ciudad de Córdoba y se estudiaron los mismos en base al análisis de fragmentos de discurso según Jäger(18).

Existen diferentes formas de investigar los discursos; la sociología, en su aproximación tradicional al discurso, parece haberse concentrado en ciertas líneas de investigación que tratan de reflejar las características sociales de una comunicación dejando de lado la noción de discurso en sí. El análisis de los textos que se centró en un análisis léxico dominado por la cuantificación de las variables analizadas, habitualmente desde técnicas de análisis de contenido, generalmente desde una perspectiva cuantitativa. Otra forma de aproximación al fenómeno de lo discursivo, desde un ámbito social, es el Análisis Crítico del Discurso cuyo objetivo fue estudiar el discurso como práctica social, prestando especial interés al 
contexto de uso del mismo, y a la relación entre textos y estructuras sociales.

El presente trabajo focalizó en el análisis del discurso basado en la propuesta de Jäger, el cual entiende a los textos como producto de la actuación social de los individuos dentro de un contexto socio-histórico. El trabajo de Sigfried Jäger, presenta un análisis crítico del discurso basado en aspectos de la obra de Michel Foucault (18).

Jäger define a los discursos como "flujo de conocimientos a través del tiempo", por lo tanto, en un análisis del discurso o de sus fragmentos debe incluirse el factor tiempo (18). Este trabajo no pretende explorar todas las ramas del discurso. Interesa el fragmento aislado, tradicionalmente llamado "texto", pero visualizando la relevancia social y política de los textos (18).

Según Jäger un análisis debe sobrepasar la observación puramente lingüística e incluir en igual medida elementos visuales, no verbales y gráficos.

Jäger define cinco pasos esenciales para el análisis de fragmentos de discurso:

1 Marco Institucional: contexto institucional.

2 Superficie del texto: unidades de sentido, temas tratados.

3 Medios lingüísticos retóricos: microanálisis lingüístico, estrategias de argumentación, vocabulario, actores, referencias.

4 Enunciados de contenido lógico: imagen del hombre, concepción de sociedad.

5 Interpretación: representación sistemática del fragmento seleccionado (18).

Los programas y planes de salud analizados fueron:

A nivel nacional:Plan Nacer,Programa Maternidad e Infancia

A nivel provincial: Plan Nacer Córdoba,Programa Maternidad e Infancia Córdoba

A nivel municipal:Programa Control de Crecimiento y Desarrollo, Programa Integral de Atención de Embarazo y Puerperio.

Se trabajó a nivel temático y a nivel contextual, como se observa en el siguiente cuadro:

\begin{tabular}{|c|c|}
\hline$\frac{\text { Aspecto temático-Nivel semántico- }}{\text { Análisis de contenido: }}$ & $\frac{\text { Aspecto contextual - Nivel pragmático- }}{\text { Análisis del marco institucional: }}$ \\
\hline Determinación del tema & $\begin{array}{c}\text { Identificación de lugar, fecha, emisión, } \\
\text { público destinatario }\end{array}$ \\
\hline Hallazgo de palabras claves & $\begin{array}{c}\text { Análisis de la posición ideológica del autor. } \\
\text { (repeticiones de significados semánticos). }\end{array}$ \\
\hline $\begin{array}{c}\text { Descubrimiento de presuposiciones } \\
\text { referencias explícitas o implícitas a } \\
\text { conocimientos previos, a valores y a normas } \\
\text { culturales). }\end{array}$ & $\begin{array}{c}\text { Lonanalización de referencias a } \\
\text { acontecimientos discursivos. } \\
\text { política, económica y social actual. }\end{array}$ \\
\hline $\begin{array}{c}\text { Identificación de elementos intertextuales } \\
\text { (citas, referencias, incorporación de párrafos } \\
\text { de otros textos). }\end{array}$ & $\begin{array}{c}\text { Explicitación de la intención y de la } \\
\text { búsqueda de efecto. }\end{array}$ \\
\hline
\end{tabular}

\section{Resultados}

Los programas y planes analizados fueron Plan Nacer, Programa Maternidad en Infancia, ambos bajo la órbita del Ministerio de Salud Nacional y Provincial; y el Programa Atención Integral del Embarazo y Control de Crecimiento y Desarrollo Infantil, estos programas bajo la Dirección de Atención Primaria de la Salud, de la Municipalidad de Córdoba. 


\section{Programas y Planes de Salud Nacionales y Provinciales Plan Nacer ${ }^{3}$}

El Plan Nacer es un programa federal del Ministerio de Salud de la Nación que invierte recursos en salud para mejorar la cobertura de salud y la calidad de atención de las mujeres embarazadas, puérperas y de los niños/as menores de 6 años que no tienen obra social. Se inició en el año 2005 en las provincias del NEA y NOA y en 2007 se extendió a todo el país.

El programa se distingue por desarrollar seguros públicos de salud para la población materno- infantil sin obra social y ser un modelo de financiamiento basado en resultados, que se propone promover un cambio cultural en la visión y gestión de la salud que priorice la estrategia de la atención primaria de la salud (APS), la eficacia del sistema y la utilización efectiva de los servicios de salud por parte de la población.

Es una herramienta de gestión que fortalece al sector salud para garantizar la seguridad de la atención, existen otros programas con los que está asociado: Asignación Universal por Hijo, Control de Crecimiento y Desarrollo y Control de Embarazo. Esta herramienta se efectiviza a través de convenios que se firman entre el estado nacional y los estados provinciales. El convenio está relacionado con el nivel presupuestario provincial porque no debe disminuir su presupuesto por recibir el plan nacer, hasta que llega un punto en que la provincia se hace cargo del financiamiento del plan.

El Plan Nacer a nivel nacional, eleva directrices a las provincias quienes son las encargadas de su ejecución.

La asignación de roles dentro del Plan Nacer es un aspecto central y mantiene una clara lógica dentro de los límites de la estructura federal del país. En este sentido los roles y responsabilidades quedan delimitadas de la siguiente manera:

- La Nación preserva para sí un rol normativo, de financiamiento y de supervisión.

- Las Provincias tienen un rol ejecutivo llevando adelante los Seguros Provinciales de

Salud para promover una cobertura efectiva (integral, oportuna y de calidad).

Los establecimientos de la red pública -centros integradores comunitarios (CIC), unidades sanitarias, dispensarios, salitas, centros de atención primaria de la salud (CAPS), maternidades u hospitales- actúan realizando las prestaciones, cobrando por ellas y utilizando los recursos para mejorar los servicios de salud brindados a la comunidad.

Se presenta como un texto argumentativo, con significados claros respecto de cobertura y calidad de atención. Se reconoce explícitamente la situación de salud materno infantil, de valores y normas culturales en relación a la calidad de atención, pero no hace referencia explícita a las prácticas de cuidado por parte de la población. El cuidado es considerado condición previa al cumplimiento de los derechos de niños/niñas. Si bien a nivel de las trazadoras sanitarias, no se incorporan aspectos que aporten en el asesoramiento sobre salud y nutrición para los cuidadores, los mismos están previstos a nivel de ejecución programática en la prestación consejos para los padres.

\section{Programa Maternidad e Infancia ${ }^{4}$}

La Dirección Nacional de Maternidad e Infancia se encuentra en la actualidad, dentro del ámbito de la Secretaría de Promoción y Programas Sanitarios, Subsecretaría de Salud Comunitaria, del Ministerio de Salud de la Nación. Es el órgano de formulación y aplicación de las políticas materno-infantiles del Estado Nacional. Allí se desarrolla el Programa

\footnotetext{
${ }^{3}$ Se puede acceder al Plan Nacer a través del sitio web: www.plannacer.msal.gov.ar y en Córdoba a través de http://www.cba.gov.ar/plan-nacerministerio-de-salud

${ }^{4}$ Se puede acceder al programa maternidad e infancia a través de la página web: http://www.msal.gov.ar/promin/ en Córdoba a través de http://www. cba.gov.ar/direccion-de-maternidad-e-infancia/.
} 
Materno Infantil cuya función es la asistencia técnica y financiera a las provincias argentinas. Para la implementación de las políticas de salud materno infantil fueron fijados tres ejes estratégicos prioritarios: Salud Perinatal, Salud Integral del Niño y Salud Integral en la Adolescencia.

Como dirección nacional, eleva sus directrices a las direcciones de maternidad e infancia provinciales.

El sitio web está estructurado en una portada inicial que explica "quienes somos" y 4 solapas (Salud perinatal, Salud integral del niño, Nutrición, contacto); y una serie de accesos directos (curvas de monitoreo de crecimiento, el resultado de la Encuesta Nacional de Nutrición y Salud (ENNyS), la descarga de programas, el Plan Nacer)

La Dirección de Maternidad e Infancia fue creada en 1936, en sus inicios dependió del Ministerio del Interior, ya que aún no había sido creado el Ministerio de Salud, que se creó 10 años después. A principios de los ' 90 sus acciones pasan a formar parte del Compromiso Mundial a Favor de la Infancia, suscrito por Argentina y traducido a Metas en Favor de la Madre y el Niño. Se presenta como un texto expositivo, con significados claros respecto de asistencia técnica (apoyo a los equipos provinciales), integralidad de atención (acompañando a las familias en la crianza), transformación del modelo de atención (acciones centradas en el apoyo y sostén de la familia). Se reconoce explícitamente la situación de salud materno-infantil. El documento expresa que su estrategia se basa en los derechos ciudadanos presente en el "Compromiso Nacional en favor de la Madre y el Niño". A nivel discursivo el apoyo a las familias en el cuidado se vincula a la capacitación y evaluación de los equipos de salud, no haciendo referencia directa sobre las prácticas de cuidado nutricional.

\section{Programas de Salud Municipales}

Programa Atención Integral del Embarazo ${ }^{5}$

En la ciudad de Córdoba, el primer nivel de atención de salud es administrada por la Secretaría de Salud y Ambiente de la Municipalidad que asiste a la población en cada uno de sus Centros de Atención. El programa atención del embarazo se efectúa en estos centros. Está destinado a brindar a la embarazada atención integral desde la concepción hasta el puerperio. Sus objetivos son: Brindar atención a la demanda espontánea de embarazadas y puérperas; Extender la cobertura de atención a la totalidad de la población de embarazadas y puérperas del Área Programática que no reciben atención médica; Evaluar el estado nutricional de la embarazada; Asegurar el $100 \%$ de los partos Institucionales; Reducir la morbimortalidad materno-fetal.

Para el logro de sus objetivos, el programa plantea una serie de actividades:

- $\quad$ Captación de embarazadas y puérperas que concurren al Centro de Salud.

- Búsqueda activa de embarazadas y puérperas en el Área Programática.

- Seguimiento clínico, social, y educativo a las embarazadas y puérperas de acuerdo a normas.

- Derivación de embarazadas y puérperas a otros niveles de atención según grado de riesgo.

- Derivación de embarazadas para el parto institucional.

- $\quad$ Educación para la Salud individual y colectiva (19).

El documento, creado en 2010, es de tipo expositivo, dirigido a los equipos de salud. Brinda una serie de métodos y técnicas a emplear en cada control prenatal, aparece repetición de términos como: alimentación (evaluación de la alimentación, educación alimentaria, alimentos fuente de nutrientes, alimentación del recién nacido); higiene (higiene personal, higiene de mamas, higiene personal, higiene bucal, higiene del recién nacido); cuidado (cuidado del embarazo, cuidado de mamas, cuidado del recién nacido, cuidado de la boca).

\footnotetext{
${ }^{5}$ Las citas son textuales tal como aparecen en el documento
} 
Sugiere considerar a la familia bajo su faz dinámica propia, en la que influyen factores socioeconómicos, culturales y de salud individual que incide en la salud familiar.El Programa Atención de Embarazadas y Puérperas considera al embarazo como un proceso natural y normal, que está influenciado por expectativas y miedos, sinembargo, no se incorporan indicadores que aporten en el asesoramiento sobre estos aspectos para las embarazadas.

\section{Programa Control de Crecimiento y Desarrollo del Niño ${ }^{6}$}

El Programa Control de Crecimiento y Desarrollo (CCyD) es un programa de primer nivel de atención de la municipalidad de Córdoba. Está destinado a promover la salud física, mental y emocional de la población de 0 a 6 años del Área Capital. La/os niña/os que se encuentran bajo programa, tienen un seguimiento continuo de su crecimiento y desarrollo en todas las etapas: desde el control de la madre, embarazo y puerperio, el nacimiento y hasta los 6 años y en la escuela, si asiste a un establecimiento municipal o si se atiende usualmente en algún centro de salud. Los ejes de acción que orientan dicho programa son:

- La promoción de la salud física, mental y emocional de la población de 0 a 6 años del Área Capital.

- La prevención de alteraciones en el proceso de crecimiento y desarrollo, contribuyendo así disminuir las tasas de morbilidad y mortalidad infantil, mediante la captación precoz y seguimiento de la/os niña/os bajo programa, la vigilancia activa de su evolución y la introducción de la estimulación temprana.

- La satisfacción de la demanda de atención de crecimiento y desarrollo, como de patologías de niños que concurren espontáneamente a los centros de salud, extendiendo la cobertura a la población expuesta a riesgo que no recibe atención en otros niveles o subsectores de atención médica (público, privado, obras sociales).

- La difusión de la importancia del control de crecimiento y desarrollo del niño en el Área Programática.

- El conocimiento de la situación de salud de la población infantil bajo programa (20).

El documento fue realizado en 2010, es la continuación de diversas revisiones del programa desde 1999. Está destinado a promover la salud física, mental y emocional de la población de 0 a 6 años del Área Capital. La estrategia de argumentación utilizada es la de concesión de la comunidad, que remite al reconocimiento de ciertos puntos de vista del otro como válidos. El documento gira en torno a las normas de atención del equipo de salud, determinando las actividades y el enfoque de atención que debe tener el equipo de salud. El documento se posiciona desde un enfoque de salud integral del niño; haciendo referencia al modelo extendido de cuidados, propuesto por Unicef y OMS. El documento considera una serie de características de las personas y de la comunidad, que deben tenerse en cuenta, ya que se definen como riesgo para la salud del niño. En cuanto a la alimentación, el documento reconoce las diferencias culturales, económicas y sociales en torno a la elección alimentaria. En las actividades propuestas al equipo de salud se encuentra la de creación de espacios grupales de reflexión-acción; actividades de capacitación con metoodología participativa; coordinación entre instituciones; educación para la salud, además de la actividad asistencial de control en consultorio. A nivel discursivo se visualiza un posicionamiento normativo hacia el equipo de salud, en relación a las actividades se observa la incorporación de una mirada de salud integral con acciones que van más allá de lo asistencial, puertas afuera del consultorio.

${ }^{6}$ Las citas son textuales, tal como aparecen en el documento. 
El presente estudio halló que en los documentos analizados se incorpora la perspectiva de derechos. La perspectiva de los derechos es un enfoque global de intervención en donde la política social hace hincapié en los mecanismos y dispositivos de la Seguridad Social que se efectiviza en quienes se encuentran en el mercado laboral formal (asignaciones familiares, seguro de desempleo, obras sociales, vivienda, jubilaciones), la Salud y Educación públicas que revisten un carácter universal, y la Asistencia Social para las poblaciones que no acceden al mercado laboral (transferencia de ingresos, promoción de la economía social (subsidio y crédito) y política alimentaria) (21). Coincide con autores como Arriagada quien realiza un análisis de la situación de salud y los programas de salud en la región desde una perspectiva de derechos, y expresa que, en relación con la medicina comunitaria, en países como Argentina, Nicaragua, Panamá se ha pretendido extender el sistema sanitario a la intimidad de los hogares, permitiendo conocer la realidad social y sanitaria de la población, y mejorando la comunicación del individuo y su familia, su saber y su cultura con la medicina científica (22).

El presente estudio encontró que los programas municipales no hacen mención a los programas nacionales y provinciales, pero sí, todos acuerdan con normas internacionales, lo cual coincide con el estudio de Arraigada el cual indica que, en Argentina, la fragmentación del sistema en subsectores (público, de la seguridad social y privado), en jurisdicciones (nacional, provincial y municipal) y en niveles de atención (primer, segundo y tercer nivel), ha generado un uso ineficiente de los recursos disponibles al duplicar innecesariamente ofertas y servicios, y, por tanto, los gastos.

El Plan Nacer se visualiza como un programa que intenta integrar los demás programas; no sólo desde el discurso, sino también en su ejecución, concibiendo al sistema de salud como algo integrado y que necesariamente debe actuar en consonancia con los demás programas de salud que se implementan.

También como parte de los resultados se identificó que los documentos no indican de qué manera medir sus objetivos, coincidiendo con Almeida y Atalah quienes reconocen la importancia del apoyo al cuidado en los primeros años de vida, pero indican que la mayor parte de los sistemas de información estadística aportan datos sobre el número de controles realizados y de la población bajo control y prácticamente no existe información sobre el grado de cumplimiento de las diferentes actividades definidas en las normas, ni menos sobre su calidad $(23,24)$.

El plan nacer, a diferencia de los otros planes y programas de salud analizados, incorpora trazadoras sanitarias,Si bien a nivel de las trazadoras sanitarias, no se incorporan aspectos que aporten en el asesoramiento sobre salud y nutrición para los cuidadores, si están previstos a nivel de ejecución programática en la prestación "consejos para los padres". Estos aspectos se vinculan con la visión de APS ampliada Este resultado coincide con un análisis del Plan Nacer en el Informe N³ del 2012 del Documento Series Políticas Públicas, concluyendo que el mismo puede ayudar al logro de los objetivos del milenio, específicamente en lo referido a salud materna y mortalidad infantil (25).

Otro de los resultados obtenidos refiere que la mujer es vista desde su rol reproductivomaterno. Este resultado coincide con un estudio sobre políticas y programas de salud en América Latina, publicado por la CEPAL que examinó la situación de la salud y los programas de atención de los países latinoamericanos, sobre la base de la información obtenida de las respuestas de los ministerios de salud de 17 países a la encuesta que sobre el tema efectuó CEPAL (22).

Otros autores coinciden con los resultados de este trabajo en la mirada de la mujer desde su rol reproductivo, como el trabajo de Arriagada, al preguntar sobre los grupos de población con mayor impacto de los problemas de atención de salud, sólo en el caso de Perú se menciona a la población femenina como uno de los grupos en los cuales los problemas de 
atención de salud inciden con mayor intensidad, ya que registran una más alta carga de enfermedades (22).

Esta mirada, también es observado por Gómez, quien reconoce una inexistencia en América Latina, de políticas públicas sólidas dirigidas según grupos de edad y sexo, continuando así, el tratamiento de las mujeres centrado en su papel reproductivo-materno, en menoscabo del examen y ponderación del amplio rango de necesidades, riegos y contribuciones ligados a los múltiples papeles que la mujer cumple en las distintas etapas de su vida $(22,26)$.

En el presente estudio se observa que los documentos analizados pretenden incorporar la comunicación entre los sujetos y los equipos de salud; hay una intención de centrar las acciones en la comunidad; pero la falta de coordinación y articulación entre los distintos subsectores proveedores de servicios de salud (público, seguridad social y privado) generan una heterogénea oferta de coberturas, que impide la conformación de una visión compartida de los sistemas de salud y atenta contra el uso eficiente de recursos y el logro de niveles aceptables de equidad en el acceso y la utilización de los servicios, coincidiendo con Arriagada. (22).

\section{Conclusión}

En relación a los programas y planes analizados, se puede observar que en la Argentina las políticas de salud en torno a los cuidados se conciben desde el régimen familista.

A nivel de los documentos se observa que, si bien el cuidado sigue siendo responsabilidad de las familias, hay un reconocimiento de que es necesario empoderar a las mismas para el ejercicio del derecho al cuidado, en todos los programas y planes se proponen actividades en un marco de diálogo entre los efectores y cuidadores.

Si bien en los documentos se hace referencia al cuidado compartido, éstos se encuentran en el contexto de una política familista, donde la responsabilidad principal del bienestar corresponde a las familias y a las mujeres en las redes de parentesco. Son las cuidadoras de la familia, ellas se encuentran condicionadas por la cultura, la cual atraviesa un conjunto de comportamientos, creencias y actitudes con respecto al cuidado tanto de los otros como el de ellas.

\section{Bibliografía}

1. Kraft, M., Furlong, S. Public Policy: Politics, Analysis and Alternatives, 2nd ed., Washington, DC: CQ Press; 2007, p 5.

2. Castro Sánchez AE y Evangelista Martínez E. «Investigación en Políticas Sociales» en, Revista Trabajo Social. Revista de la ENTS-UNAM, Número 21 Desarrollo Social, septiembre diciembre 1998. [En línea] Disponible en: http://www.ubiobio. $\mathrm{cl} / \mathrm{cps} /$ ponencia/doc/p8.3.htm

3. Grassi E. Política y problemas sociales en la sociedad neoliberal La otra década infame (1), Buenos Aires: Espacio, 2003.

4. Maingon T. Política Social en Venezuela 1999-2003. Cuadernos del CENDES, Año $21 N^{\circ}$ 55, Tercera Época, Enero-Abril 2004. [En línea]Disponible en: http://www. redalyc.org/html/403/40305504/

5. García Puente MJ. La evaluación de políticas públicas y sociales. Un análisis entorno a los actores. En ponencia presentada en el VI Congreso Nacional de Ciencia Política. Rosario 1994. P 5-8.

6. Álvarez MF y otros. Centro de Estudios de Población y Desarrollo (CEPyD). Ensayos demográficos sociales. N1. Características Sociodemográficas de la población. Provincia de Córdoba. Ciudad de Córdoba, 2004.

7. Paganini JM, Etchegoyen G, Arrondo F. Guía para el análisis y evaluación de los efectores de primer nivel. - 1a ed. - La Plata: Univ. Nacional de La Plata, 200931 p. 
8. Esquivel V, Faur E, Jelín E. Hacia la conceptualización del cuidado: familia, mercado y Estado. Las LógCuidInfant Entre Las Fam El Estado El Merc. 2012;11-43.

9. Aguirre R. "Los cuidados familiares como problema público y objeto de políticas" En Arriagada, I. Familias y políticas públicas en América Latina: Una historia de desencuentros, Comisión Económica para América Latina y el Caribe (CEPAL), Santiago de Chile.2007

10. Letablier M.T. "El trabajo de 'cuidados' y su conceptualización en Europa". En: Prieto (ed.) (ed.) Trabajo, género y tiempo social. Hacer/Complutense, Madrid. 2007, p 64-84.

11. Jelin E. Pan y afectos. La transformación de las familias, Fondo de Cultura Económica, Buenos Aires. 2010

12. Draibe S, Riesco M. Estado de bienestar, desarrollo económico y ciudadanía: algunas lecciones de la literatura contemporánea. CEPAL. Serie Estudios y Perspectivas, ${ }^{\circ}$ 55. México 2006.

13. Ley Nacional 26.061 Boletín Oficial. Argentina, 21 de octubre de 2005.

14. Engle, P., Menom, P., Haddad, L. Care and nutrition. Concepts and measurement. [Enlineal]. FCND Discussion Paper No 18. IFPRI. August 1996. Disponible en la URL:http://econpapers.repec.org/paper/fprfcnddp/18.htm

15. Batthyany, k. Genta, N. Perrotta, V. La población uruguaya y el cuidado: Persistencias de un mandato de género. Encuesta nacional sobre representaciones sociales del cuidado: Principales resultados. 2012. [En línea] Disponible en: http://www.cepal. org/publicaciones/xml/6/48256/SLapoblacionUruguayayelcuidado.pdf.

16. Pautassi L "El cuidado como cuestión social desde un enfoque de derechos". Serie Mujer y Desarrollo. CEPAL. Unidad Mujer y Desarrollo. Octubre 2007.

17. Mendizábal N. Capítulo 2. Los componentes del diseño flexible en investigación cualitativa. En: Vasilachis de Gialdino I. Estrategias de Investigación Cualitativa. Editorial Gredisa. Biblioteca de Educación. Noviembre 2006.

18. Hennecke A. El discurso como flujo de conocimientos a través del tiempo: reflexiones sobre la relación entre discurso y realidad. Córdoba. Argentina. Comunicarte, 2004.

19. Municipalidad de Córdoba. Secretaria de Salud. Dirección de atención primaria a la salud. Programa Atención de Embarazadas y Puérperas. [Monografía DVD]. Comisión de revisión 2004.

20. Municipalidad de Córdoba. Secretaria de Salud. Dirección de atención primaria a la salud. Programa de control de crecimiento y desarrollo del niño. [Monografía DVD]. Tercera comisión de revisión 2010.

21. Tirenni J. La política social argentina ante los desafíos de un Estado inclusivo (2003-2013) [Social Policy in ArgentinafacingtheChallenges of an Inclusive State (2003-2013)]. Estado y Políticas Públicas, 2013, vol. 1, no 1, p. 123-140.

22. Arriagada I, Aranda V y Miranda F. Políticas y programas de salud en América Latina: Problemas y propuestas. Vol. 114. UnitedNationsPublications, 2006

23. Atalah S E, Pizarro V M. Análisis de las mejores prácticas recientes en América Latina en políticas de nutrición para cumplir con los Objetivos de Desarrollo del Milenio: informe de consultoría Versión preliminar [Internet]. CEPAL; 2008 [cited 2014 Jul 27]. Available from: http://bases.bireme.br/cgi-bin/wxislind.exe/iah/ online/?IsisScript $=\mathrm{iah} / \mathrm{iah} . \mathrm{xis} \& \mathrm{src}=$ google\&base=REPIDISCA\&lang=p\&nextActi on=lnk\&exprSearch $=179257 \&$ indexSearch $=$ ID

24. Almeida, C., Bazzani, R. y Pittman, P. (Org.). Investigación sobre reformas del sector salud en América Latina y el Caribe. Reflexiones sobre sus contribuciones al desarrollo de políticas. Washington D.C.: Organización Panamericana de la Salud. 2001.

25. Fundación Contemporánea. Políticas Públicas relacionadas con la salud Materno infantil. Serie Políticas públicas. Informe N³. Agosto 2012

26. Gómez E. La salud y las mujeres en América Latina y el Caribe: viejos problemas y nuevos enfoques $=$ Health and women in Latin America and the Caribbean: old issues and new approaches. 1997. 